\title{
Evaluation of an ultraviolet LED trap for catching Anopheles and Culex mosquitoes in south-eastern Tanzania
}

\author{
Emmanuel P. Mwanga ${ }^{1 *}\left(\mathbb{D}\right.$, Halfan S. Ngowo ${ }^{1,2}$, Salum A. Mapua', Arnold S. Mmbando', \\ Emmanuel W. Kaindoa ${ }^{1,3}$, Khamis Kifungo ${ }^{1}$ and Fredros O. Okumu ${ }^{1,2,3}$
}

\begin{abstract}
Background: Improved surveillance techniques are required to accelerate efforts against major arthropod-borne diseases such as malaria, dengue, filariasis, Zika and yellow-fever. Light-emitting diodes (LEDs) are increasingly used in mosquito traps because they improve energy efficiency and battery longevity relative to incandescent bulbs. This study evaluated the efficacy of a new ultraviolet LED trap (Mosclean) against standard mosquito collection methods.

Methods: The study was conducted in controlled semi-field settings and in field conditions in rural south-eastern Tanzania. The Mosclean trap was compared to commonly used techniques, namely CDC-light traps, human landing catches (HLCs), BG-Sentinel traps and Suna traps.

Results: When simultaneously placed inside the same semi-field chamber, the Mosclean trap caught twice as many Anopheles arabiensis as the CDC-light trap, and equal numbers to HLCs. Similar results were obtained when traps were tested individually in the chambers. Under field settings, Mosclean traps caught equal numbers of An. arabiensis and twice as many Culex mosquitoes as CDC-light traps. It was also better at trapping malaria vectors compared to both Suna and BG-Sentinel traps, and was more efficient in collecting mosquitoes indoors than outdoors. The majority of An. arabiensis females caught by Mosclean traps were parous (63.6\%) and inseminated (89.8\%). In comparison, the females caught by CDC-light traps were $43.9 \%$ parous and $92.8 \%$ inseminated.

Conclusions: The UV LED trap (Mosclean trap) was efficacious for sampling Anopheles and Culex mosquitoes. Its efficacy was comparable to and in some instances better than traps commonly used for vector surveillance. The Mosclean trap was more productive in sampling mosquitoes indoors compared to outdoors. The trap can be used indoors near human-occupied nets, or outdoors, in which case additional $\mathrm{CO}_{2}$ improves catches. We conclude that this trap may have potential for mosquito surveillance. However, we recommend additional field tests to validate these findings in multiple settings and to assess the potential of LEDs to attract non-target organisms, especially outdoors.
\end{abstract}

Keywords: Malaria, An. arabiensis, An. funestus, Culex spp., Mosclean trap, Ifakara Health Institute, Light-emitting diodes (LEDs)

\footnotetext{
*Correspondence: emwanga@ihi.or.tz

${ }^{1}$ Environmental Health and Ecological Sciences Department, Ifakara

Health Institute, Morogoro, Tanzania

Full list of author information is available at the end of the article
} 


\section{Background}

The World Health Organization, through its Global Technical Strategy for Malaria Elimination (GTS) [1] and Global Vector Control Response initiative (GVCR) [2], has called for strengthening and integration of surveillance as a core component of strategies against mosquitoborne diseases. To operationalize this agenda, endemic countries need low-cost and scalable monitoring tools, as well as a simplified set of indicators for surveillance. For malaria and other vector-bone diseases, surveillance plays a major role in: (i) tracking transmission; (ii) assessing susceptibility of vectors to interventions; (iii) measuring receptivity in specific locations; and (iv) predicting disease outbreaks $[3,4]$. Various traps have been developed and used for mosquito sampling and surveillance, often for experimental studies but also for programmatic purposes [5-7]. Some of the traps have also been considered as control intervention when used as mosquito trapping [8-10]. A major setback for many of the existing techniques is poor scalability due to their physical structure and cost.

The human landing catch (HLC) is the most direct and scalable method for measuring human biting rates [11] and is regarded as the gold standard for collecting hostseeking mosquitoes [7, 12-15]. However, using humans directly as bait to collect mosquitoes has multiple limitations. For example, this technique: (i) is expensive in large-scale operations; (ii) exposes humans to mosquito bites and thus increases the risk of infections in field settings; (iii) is labor-intensive; and (iv) needs close supervision, high skills and motivation [16]. To address these shortcomings, new ways of safely carrying out HLCs have been proposed and tested, e.g. the human-baited double net (HDN) and mosquito electrocuting grid trap (MET) $[17,18]$. Another common trap is the Center for Disease Prevention and Control light trap (CDC-light trap), improved by Sudia \& Chamberlain [19] and widely used for indoor collections of host-seeking Anopheles [20, 21]. The CDC-light trap uses incandescent light bulbs, battery cells and a motor-driven fan, all of which make it expensive and difficult to maintain in many settings. Despite these challenges, the CDC-light trap is still considered one of the simplest trapping techniques, requiring only light as an attractant.

Recent developments in trapping technologies and improved understanding of mosquito olfactory systems have resulted in several new trapping devices for malaria vectors. Some of these exploit the olfactory behaviors and biting preferences of mosquitoes, e.g. BG-Sentinel trap, Suna trap, Ifakara tent trap and BG-malaria [22-24]. The BG-Sentinel trap and Suna trap are simple and portable tools which can be used to sample day-biting and nightbiting mosquitoes including Anopheles and Aedes [23, 25,
26]. Others, such as the Ifakara tent trap, allow exposurefree sampling in rural and urban settings [14, 24]. One drawback of these traps is that they usually require synthetic lures, such as Ifakara lure [27], Mbita lures (MB5) [28] or carbon dioxide gas $\left(\mathrm{CO}_{2}\right)$ to mimic human odors. Others such as the Ifakara tent trap and human-baited double net traps, are large, bulky and inconveniently require human volunteers as bait.

Light-emitting diodes (LEDs) are also increasingly being tested for arthropod trapping. Compared to standard incandescent light bulbs used in CDC-light traps, the LED technology provides: (i) higher energy efficiency; (ii) longer battery life; (iii) lower costs per unit time of trapping; (iv) options for specific wavelengths targeting different arthropod species; and (v) greater trapping efficiencies.

CDC-light traps have previously been modified by replacing the incandescent bulbs with LEDs emitting blue, green or red light to achieve higher trapping efficiencies [29-33]. For example, Costa-Neta et al. [29] showed that LED lamps emitting blue $(470 \mathrm{~nm})$ or green $(520 \mathrm{~nm})$ light consistently caught high numbers of Anopheles mosquitoes, regardless of the lunar cycles. Silva et al. [31] also showed the superiority of greenLEDs over incandescent light for catching different species of sand flies in Brazil. Elsewhere, in Egypt, red-LEDs attracted more Phlebotomus papatasi sand flies than either blue or green LEDs or incandescent bulbs [32]. Lastly, tests on woodland mosquitoes in Florida revealed differential attractiveness of species to LEDs of different colors [33].

The Mosclean trap is a simple LED trap which utilizes ultraviolet (UV) emitting diodes combined with a titanium dioxide $\left(\mathrm{TiO}_{2}\right)$ plate for photocatalytic conversion to produce $\mathrm{CO}_{2}$. The trap is created by Sensor Electronic Technology Inc. (Columbia, SC, USA) and Seoul Viosys (Gyeonggi-do, Republic of Korea), using proprietary UV LED technology (violeds ${ }^{\mathrm{TM}}$ ). It has been previously demonstrated as highly efficacious for trapping Aedes albopictus and Aedes aegypti mosquitoes in the USA [34], but to our knowledge has yet to be tested against Anopheles mosquitoes anywhere.

The aim of the present study was to evaluate the efficacy of the Mosclean trap in catching malaria vectors and other mosquitoes in south-eastern Tanzania in comparison to the CDC-light trap, BG-Sentinel trap and Suna trap.

\section{Methods \\ Description of the semi-field system and study areas for field experiments}

Experiments were conducted under both semi-field and field settings. All semi-field tests were done inside a large 


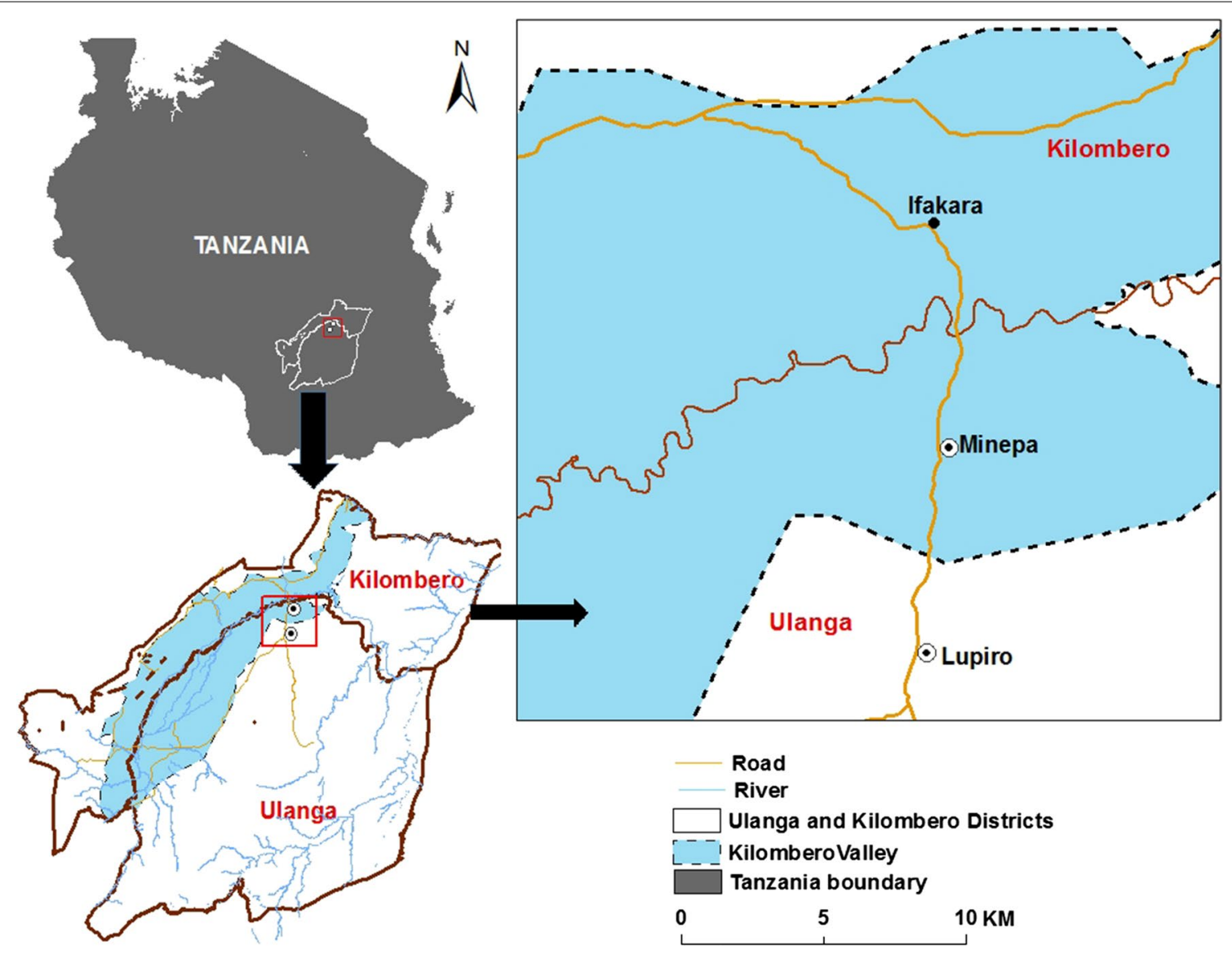

Fig. 1 Map showing study villages in Ulanga district, south-eastern Tanzania (courtesy of Alex J. Limwagu)

multi-chambered screen house $(28.8 \times 21 \mathrm{~m})$ at the Ifakara Health Institute [35], Ifakara, Tanzania. We used a compartment measuring $9.6 \times 21 \mathrm{~m}$ [35] in which there were two experimental huts with a peri-domestic area consisting of different vegetation types and pebbles, to mimic local housing and ecological setup [36].

The field tests were conducted in villages in Ulanga District, south-eastern Tanzania, approximately $20 \mathrm{~km}$ south of Ifakara town (Fig. 1). The area lies on the Kilombero flood plains between the Udzungwa Mountains in the north and the Mahenge hills to the south. The main economic activities include agriculture (mostly rice and maize cultivation), fishing and small-scale trade. Annual rainfall ranges from 1200 to $1800 \mathrm{~mm}$ and temperatures from 22 to $32.6{ }^{\circ} \mathrm{C}$. Short rains occur from November to December, while long rains occur between March and June.

\section{Mosquitoes}

The mosquitoes used were from colonies of the Ifakara strain of Anopheles arabiensis, originally established in 2009 with specimens from Lupiro village, approximately
$25 \mathrm{~km}$ south of Ifakara town. The colony is maintained at $27 \pm 2{ }^{\circ} \mathrm{C}$ and $75 \pm 10 \%$ relative humidity (RH) as previously described by Batista et al. [37]. Larvae are reared in plastic basins and fed twice a day on Tetramin ${ }^{\circledR}$ fish food (Tetra GmbH, Melle, Germany). Adults are fed on $10 \%$ glucose solution. Colony maintenance also involved ad libitum blood meals given to females via human volunteer arms. The field tests, however, targeted free-flying wild mosquitoes of different species from the study villages.

\section{Mosquito traps and lures}

The main candidate test trap was the Mosclean trap, which emits $365 \mathrm{~nm}$ UV using violeds ${ }^{\mathrm{TM}}$ technology and generates $\mathrm{CO}_{2}$ gas via a photocatalytic reaction on the $\mathrm{TiO}_{2}$ plate surfaces. The trap measures $20 \mathrm{~cm}$ (diameter) and $28.8 \mathrm{~cm}$ (height) and runs on either DC or AC current. They can operate with small portable solar cells to charge a portable power pack for use in places with no electricity.

As shown in Fig. 2a, the trap has five components: (i) roofing plate, which has a suspension tag for hanging 

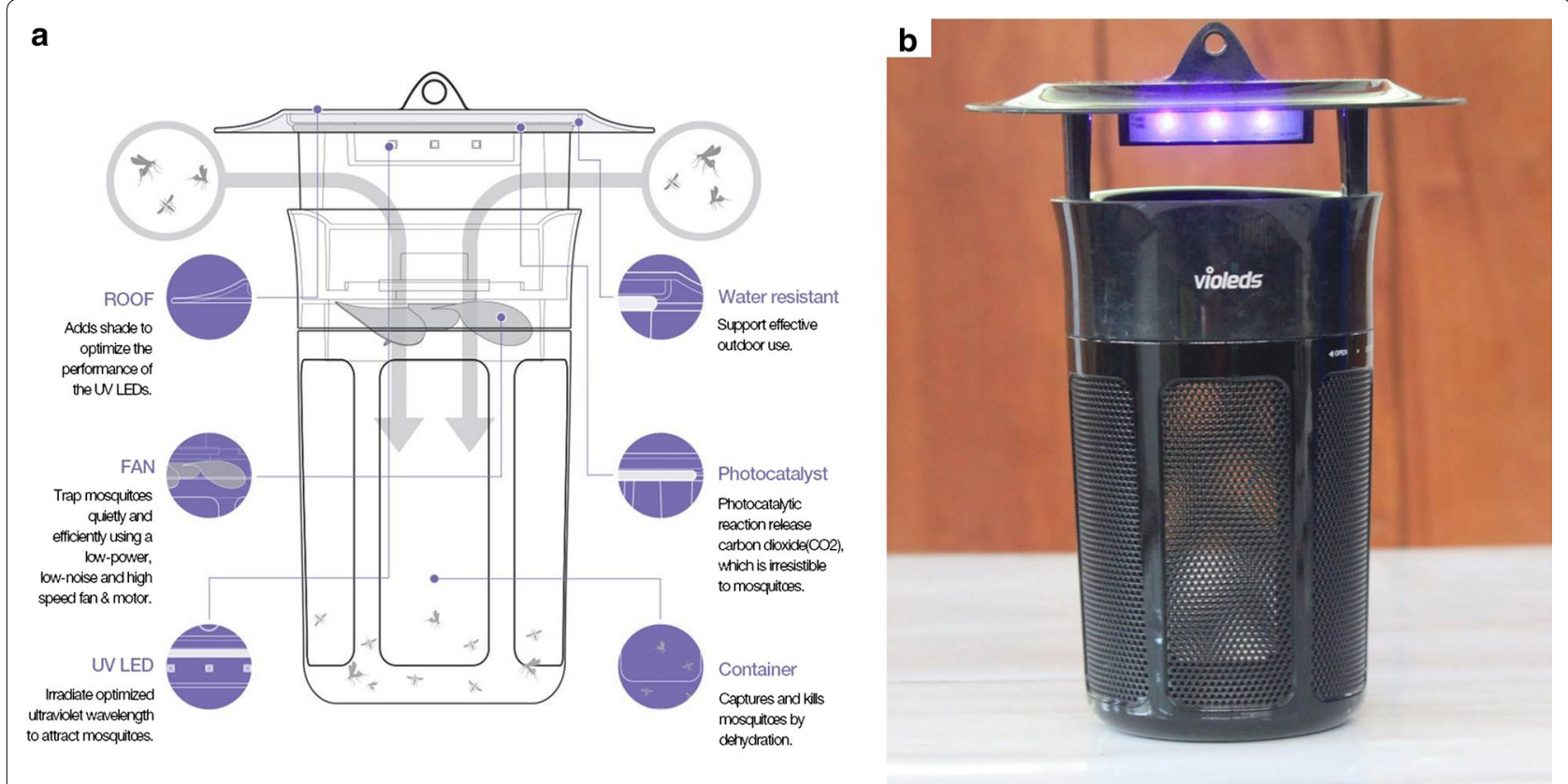

Fig. 2 a Schematic view of the Mosclean trap. b Close-up picture of the Mosclean trap. Schematics courtesy of Seoul Viosys [34]

the trap and a shade to optimize performance of the UV LEDs; (ii) a low-power, low-noise and high efficiency fan for mosquito suction; (iii) a UV LED plate, emitting ultraviolet light at a wavelength of $365 \mathrm{~nm}$ to attract mosquitoes; (iv) a container for capturing trapped mosquitoes; and (v) a photocatalyst, which according to the manufacturer releases $\mathrm{CO}_{2}$ gas to complement mosquito attraction. We did not test the $\mathrm{CO}_{2}$ production by these traps during our experiments, but instead evaluated the traps just as obtained from the manufacturer.

The trapping efficacy of Mosclean trap was compared against those of other existing traps commonly used for sampling indoor and outdoor Anopheles and culicine mosquitoes. The first trap was the CDC-light trap, widely used for trapping indoor mosquitoes $[19,20]$. The CDC-light trap uses an incandescent light bulb as the main attractant, and runs on a motorized fan powered by a battery $[19,20]$. The second was the BG-Sentinel trap (Biogents $\mathrm{GmbH}$, Regensburg, Germany) [15, 38]. The trap consists of a dark blue collapsible bucket, a white perforated lid, an intake funnel, a catch bag and a ventilator powered by a battery [23]. It is $36 \mathrm{~cm}$ in diameter and $40 \mathrm{~cm}$ in height. The last comparator trap was the Suna trap (Biogents $\mathrm{GmbH}$ ), which is $52 \mathrm{~cm}$ in diameter and $39 \mathrm{~cm}$ in height, and also operates on battery power. The Suna trap was described and optimized for the first time in western Kenya in 2014 [22] and demonstrated high efficacy against An. funestus mosquitoes in Rusinga
Island [39]. Recently it was used in Malawi to sample mosquitoes indoors and outdoors [40].

Both the BG-Sentinel and Suna traps are commonly used for sampling mosquitoes outdoors, and are often baited with proprietary BG lure, $\mathrm{CO}_{2}$ gas or other lures $[27,28,41]$. In this study, $\mathrm{CO}_{2}$ was obtained, whenever needed, from yeast-molasses fermentation, and used in the different comparator traps as detailed below. The yeast-molasses mixture was prepared 30 minutes prior to starting the experiments by mixing $40 \mathrm{~g}$ of baker's yeast and $500 \mathrm{ml}$ of molasses dissolved in $2 \mathrm{~L}$ of water, and the effluent gases channeled via plastic tubing to the traps [42]. As a standard reference, HLCs, performed by adult male volunteers, was also used in semi-field tests, but not in the field tests as the wild mosquitoes might be infectious.

\section{Study procedures}

Semi-field tests to compare trapping efficacies of the Mosclean trap, CDC-light trap, human landing catches (HLC), BG-Sentinel trap and Suna trap

The experiments were conducted inside the semi-field compartments each night from 18:00 to 06:00 h. Each night, 400 nulliparous female laboratory-reared $A n$. arabiensis aged 3-6 days and not previously blood-fed were released inside the screen house chamber [43]. The test mosquitoes were starved for $6 \mathrm{~h}$ prior to each test and were released inside the chambers $30 \mathrm{~min}$ before starting the tests to acclimatize in the environment. The huts 

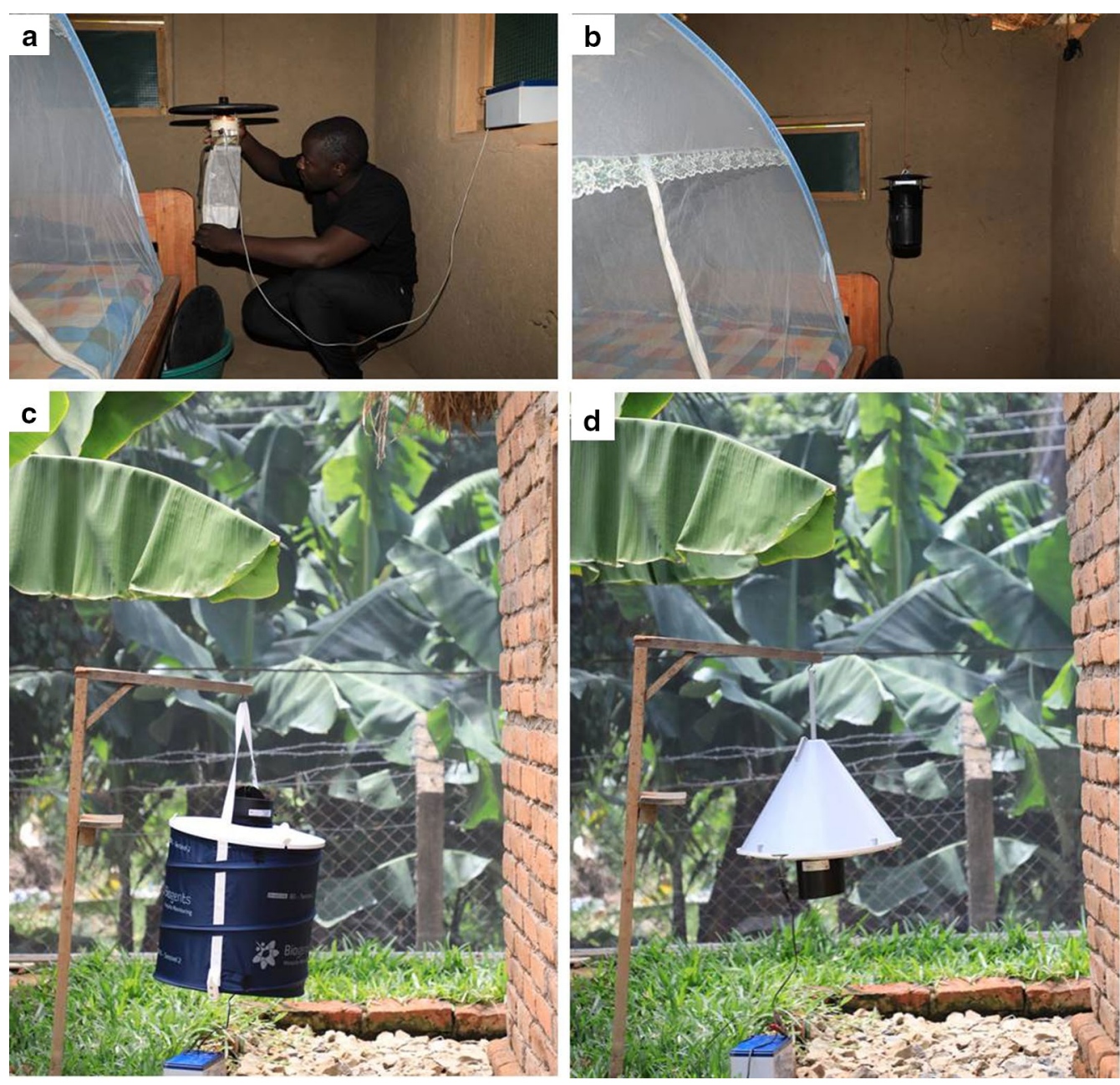

Fig. 3 Images of trap set up during semi-field experiment, a CDC-Light trap set up indoors beside a bed with a bednet. b Mosclean trap indoors beside a bed with a bednet. c BG-Sentinel trap set up outdoors. $\mathbf{d}$ Suna Trap set up outdoors

were cleaned each morning (using a Prokopack aspirator) and traps monitored for optimal functionality.

(i) Tests to compare the Mosclean trap and CDC-light trap using a human host under a bednet as bait

First, we compared the Mosclean trap against the CDClight trap for indoor trapping in tests with the two traps inside the same chamber on the same nights, such that the traps were competing for the same mosquitoes. One Mosclean trap was suspended inside one of the experimental huts, beside an untreated bednet occupied by a sleeping adult male volunteer (Fig. 3b). Similarly, one CDC-light trap was suspended inside the other hut, also beside a volunteer-occupied bednet (Fig. 3a). The traps were both $150 \mathrm{~cm}$ above ground and close to the volunteers' feet [20]. The CDC-light trap and Mosclean trap were rotated between the huts nightly to avoid positional bias. The volunteers, however, retained their positions such that their individual differential attractiveness was combined to the respective hut characteristics to constitute a single source of experimental variation. Each night, 400 female An. arabiensis mosquitoes were released from a central location inside the semi-field chamber, equidistant from the two huts. Total numbers of mosquitoes caught in each trap overnight was recorded and the traps cleaned. The test was repeated over 12 nights, each trap being in each hut six times.

(ii) Tests to compare the Mosclean trap and HLCs using human host under a bednet as bait

The second test compared the Mosclean trap and HLCs, also in a competitive manner. In one hut, a Mosclean trap was set beside a volunteer-occupied net as above (Fig. 3b), while in the second hut, HLC was conducted on the same night by an adult male volunteer. The volunteers 
sat indoors, folding their trousers to catch mosquitoes landing on their legs using mouth aspirators. This way, the two trapping methods competed for the same mosquitoes. The Mosclean trap and HLC were rotated between the huts nightly, but the volunteers stayed in the same huts. Each night, 400 female An. arabiensis mosquitoes were released from a central location inside the semi-field chamber, equidistant from the huts. Mosquito catches by either HLC or Mosclean trap were recorded and compared. The test was repeated over 12 nights, each method being in each hut six times.

(iii) Non-competitive tests of the Mosclean trap and HLCs, using human host under a bednet as bait

In the third test, the Mosclean trap was independently tested against HLC on separate nights to avoid direct competition between the traps for the same mosquitoes. The trap was set inside the first hut (randomly selected) then moved to the second hut on the second night. On the third and fourth nights, HLC was conducted inside either the first or second hut in a random order. This sequence was randomized so that the tests rounds began with either HLC or the Mosclean trap, and was also replicated six times. Each night, 400 female An. arabiensis mosquitoes were released from a central location inside the semi-field chamber. Numbers of mosquitoes caught each night by each trap type in the absence of a competing alternative were recorded compared.

(iv) Tests to directly compare the Mosclean trap against commonly used outdoor traps, i.e. Suna and BGSentinel traps

Unlike the first three tests, mosquito trapping in this experiment was conducted outdoors but within the semi-field chambers over four-night cycles using a randomized $4 \times 4$ Latin square design. Two different variants of the Mosclean trap were used, the first without any additional bait, and the second baited with additional $\mathrm{CO}_{2}$ from yeast-molasses fermentation. The Suna trap (Fig. 3d) and BG-Sentinel (Fig. 3c) were also baited with $\mathrm{CO}_{2}$ from yeast-molasses fermentation. The four trap types, standard Mosclean trap, $\mathrm{CO}_{2}$-baited Mosclean trap, $\mathrm{CO}_{2}$-baited Suna trap and $\mathrm{CO}_{2}$-baited BG-Sentinel trap were rotated nightly in a random fashion across the four outdoor locations inside the semi-field chamber over four-night cycles. A total of 600 female An. arabiensis were released each night at a central location equidistant from each trap position and the catches recorded each morning. The experiment was replicated seven times over 28 nights, during which each trap type had been to each location seven times.
Field tests to compare trapping efficacies of the Mosclean trap, CDC-light trap, BG-Sentinel trap and Suna trap

Four different field experiments were conducted as follows:

(i) Comparison of efficacies of the Mosclean and CDClight traps for sampling indoor host-seeking mosquitoes

Four houses were selected randomly in each of the study villages and recruited after obtaining consent from the household heads. In each hut, either a Mosclean trap or CDC-light trap was set beside a bed inside a room where one adult volunteer slept under bednet. Since coverage of insecticide-treated nets was $>90 \%$ in these villages, we conducted our study with these rather than untreated nets as used in the semi-field tests. Based on the trap assignment schedule, huts $1,2,3$ and 4 each received either of the two trap types. No additional lures were added to the traps. The traps (1st Mosclean trap, 2nd Mosclean trap, 1st CDC-light trap and 2nd CDC-light trap) were rotated each night between each of the houses over 12 nights, following a $4 \times 4$ Latin square design, replicated four times. The tests lasted from 18:00 to 06:00 $\mathrm{h}$ each night. Both Mosclean and CDC-light traps were suspended $150 \mathrm{~cm}$ above ground, towards the feet of the sleepers. Mosquitoes caught by each trap each night were sorted by taxa, sex and physiological status, then counted and recorded.

(ii) Comparison of the performance of the Mosclean trap when used indoors and outdoors

In studies measuring malaria vector biting risk, it is common to assess proportions of biting that occur indoors versus proportions that occur outdoors, so as to determine where the biting risk is greater and also whether interventions such as ITNs and IRS are addressing the full spectrum of exposure. Unfortunately, despite recent innovations such as the electric grid traps [17] and human-baited double net traps [18], there are no appropriate trapping systems other than HLCs that consistently and accurately assess these proportions. The Mosclean trap was therefore assessed to determine if it would be suitable for collections both indoors and outdoors. Four households were selected randomly in each village and recruited following consent from the head of household. Mosclean traps were then set either indoors or outdoors at each of the houses. When used indoors, the trap was set beside an occupied bednet. However, when outdoors, the trap was placed approximately $5 \mathrm{~m}$ from the house, but next to an adult volunteer sitting under a bednet. The Mosclean trap outdoors was set $5 \mathrm{~m}$ from the house, aiming to catch mosquitoes flying close to the human dwellings. The traps were $150 \mathrm{~cm}$ above ground, whether indoors or outdoors. Each morning the 
Table 1 Median number of female Anopheles arabiensis mosquitoes recaptured per night by Mosclean trap and other candidate traps inside the semi-field chambers in tests to compare trapping efficacies of the Mosclean trap, CDC-light trap, human landing catches (HLC), BG-Sentinel trap and Suna trap. Table also shows interquartile ranges (IQR) and relative rates (RR)

\begin{tabular}{|c|c|c|c|c|c|}
\hline Experiment & Traps tested & $\begin{array}{l}\text { Total no. of mosquitoes } \\
\text { recaptured }\end{array}$ & $\begin{array}{l}\text { Median nightly } \\
\text { catch (IQR) }\end{array}$ & $\mathrm{RR}(95 \% \mathrm{Cl})$ & $P$-value \\
\hline \multirow{2}{*}{$\begin{array}{l}\text { Competitive comparison of } \\
\text { Mosclean trap and CDC-light trap }\end{array}$} & CDC-light trap indoors & 524 & $26.5(20.5-72.75)$ & Ref & Ref \\
\hline & Mosclean trap indoors & 1102 & $100.5(87.75-118.25)$ & $2.1(0.97-4.56)$ & 0.059 \\
\hline \multirow{2}{*}{$\begin{array}{l}\text { Competitive comparison of } \\
\text { Mosclean trap and } \mathrm{HLC}\end{array}$} & HLC indoors & 1124 & $88.5(82-103)$ & Ref & Ref \\
\hline & Mosclean trap indoors & 834 & $79.5(54.75-96.25)$ & $0.74(0.51-1.07)$ & 0.113 \\
\hline \multirow{2}{*}{$\begin{array}{l}\text { Non-competitive comparison of } \\
\text { Mosclean trap and HLC }\end{array}$} & HLC indoors & 626 & $102(98.25-115.50)$ & Ref & Ref \\
\hline & Mosclean trap indoors & 817 & $97(76.75-175.75)$ & $1.1(0.67-1.82)$ & 0.708 \\
\hline \multirow{4}{*}{$\begin{array}{l}\text { Direct comparison of Mosclean trap } \\
\text { against Suna trap and BG-Sentinel } \\
\text { trap }\end{array}$} & $\begin{array}{l}\mathrm{CO}_{2} \text {-baited BG-Sentinel trap out- } \\
\text { doors }\end{array}$ & 777 & $18(5-27.25)$ & Ref & Ref \\
\hline & $\mathrm{CO}_{2}$-baited Mosclean trap outdoors & 963 & $32.5(27.75-42)$ & $1.48(0.96-2.32)$ & 0.086 \\
\hline & $\mathrm{CO}_{2}$-baited Suna trap outdoors & 1149 & $30(19.50-48)$ & $1.67(1.08-2.66)$ & 0.021 \\
\hline & Unbaited Mosclean trap outdoors & 325 & $9(4.0-13.25)$ & $0.46(0.29-0.72)$ & $<0.001$ \\
\hline
\end{tabular}

mosquitoes caught indoors and outdoors were sorted by taxa, sex and physiological status, and the numbers recorded and compared.

(iii) Tests to compare field efficacies of the Mosclean, BG-Sentinel and Suna traps outdoors

These experiments was conducted outdoors using a $4 \times 4$ Latin square design in which trap locations were randomly assigned at the start of each round of four sampling nights. Two variants of Mosclean trap were used, one supplemented with $\mathrm{CO}_{2}$ gas from yeast-molasses fermentation and another without additional $\mathrm{CO}_{2}$. Four locations were identified in each of the two villages, approximately $100 \mathrm{~m}$ apart. The four trap types, standard Mosclean trap with no additional bait, $\mathrm{CO}_{2}$-baited Mosclean trap, $\mathrm{CO}_{2}$-baited Suna trap and $\mathrm{CO}_{2}$-baited BG-Sentinel trap, were rotated nightly between the four outdoor locations over four-night cycles. Each morning, mosquitoes caught by each trap were sorted by taxa, sex and physiological status, and the numbers recorded and compared. The tests were performed in the dry season of June 2017 to February 2018.

(iv) Assessing proportions of parous and proportions of inseminated female Anopheles mosquitoes in Mosclean trap and CDC-light trap catches

Mosquitoes were sampled in four different houses in one village for 20 nights. All female $A n$. arabiensis mosquitoes collected were dissected. Similar trapping was done by CDC-light traps and the mosquitoes dissected under stereo light microscopes so that the parity and insemination rates could be compared.

The dissected ovaries or spermatheca were observed at $10 \times$ magnification under a compound microscope. Parity status was confirmed by presence of stretched ovariole tracheoles (parous females) or coiled tracheolar skeins (nulliparous females), as detailed [44]. Insemination was confirmed by observing whether the spermatheca were filled or unfilled [45].

\section{Data analysis}

Data were analyzed using open source statistical software, R v.3.5.0 [46]. The efficacy of the Mosclean trap was compared to those of the other traps by fitting generalized linear mixed modes (GLMM) using the package lme4 [47]. The number of mosquitoes of different species was modelled following negative binomial distributions, with trap type as the main effect. Experimental days and hut ID were included as a random term to account for unexplained variations within days and huts. Graphs were created in the package ggplot2 [48]. Logistic regression was used to assess the parity and insemination rates between the CDC-light trap and Mosclean trap. A likelihood ratio test was used to check the effect of random effect.

\section{Results}

Results of semi-field tests to compare trapping efficacies of the CDC-light trap, human landing catches (HLC), BG-Sentinel and Suna trap Tests to compare the Mosclean trap against the CDC-light trap and $H L C$

Results for these tests, including nightly mosquito counts and statistical parameter estimates are summarized in Table 1. Additional information is provided in Additional file 1: Figure S1. When placed simultaneously inside the semi-field chambers on the same nights, the Mosclean trap caught twice as many An. arabiensis mosquitoes as the CDC-light trap [relative rates (RR) and 95\% CI: 2.1 (0.97-4.56)], although the difference was not statistically significant $(P=0.059)$. There was also no statistically 
Table 2 Median number of female Anopheles arabiensis mosquitoes recaptured per night by Mosclean trap and other candidate traps in rural Tanzanian villages during tests to compare trapping efficacies of the Mosclean trap, CDC-light trap, BG-Sentinel trap and Suna trap. Table also shows interquartile ranges (IQR) and relative rates (RR)

\begin{tabular}{|c|c|c|c|c|c|c|}
\hline Experiment & Mosquito species & Trapping methods tested & $\begin{array}{l}\text { Total no. of mosquitoes } \\
\text { collected }\end{array}$ & $\begin{array}{l}\text { Median nightly } \\
\text { catch (IQR) }\end{array}$ & $\mathrm{RR}(95 \% \mathrm{Cl})$ & $P$-value \\
\hline \multirow{2}{*}{$\begin{array}{l}\text { CDC-light trap vs Mosclean } \\
\text { trap; indoors }\end{array}$} & \multirow[t]{2}{*}{ An. arabiensis } & CDC-light trap & 5336 & $17(5-50)$ & Ref & Ref \\
\hline & & Mosclean trap & 6291 & $21(7.75-52.25)$ & $1.18(0.89-1.56)$ & 0.242 \\
\hline \multirow{2}{*}{$\begin{array}{l}\text { CDC-light trap vs Mosclean } \\
\text { trap; indoors }\end{array}$} & \multirow[t]{2}{*}{ An. funestus } & CDC-light trap & 229 & $0(0-2)$ & Ref & Ref \\
\hline & & Mosclean trap & 147 & $0(0-1)$ & $0.62(0.43-0.89)$ & 0.009 \\
\hline \multirow{2}{*}{$\begin{array}{l}\text { CDC-light trap vs Mosclean } \\
\text { trap; indoors }\end{array}$} & \multirow[t]{2}{*}{ Culex spp. } & CDC-light trap & 9653 & $40(15-96.25)$ & Ref & Ref \\
\hline & & Mosclean trap & 22616 & 75 (33.75-163.75) & $2.18(1.72-2.77)$ & $<0.001$ \\
\hline \multirow{2}{*}{$\begin{array}{l}\text { Mosclean trap indoors vs } \\
\text { Mosclean trap outdoors }\end{array}$} & \multirow[t]{2}{*}{ An. arabiensis } & Mosclean trap outdoor & 240 & $6(2-11)$ & Ref & Ref \\
\hline & & Mosclean trap indoor & 291 & $10(2.75-16.75)$ & $1.35(0.83-2.22)$ & 0.229 \\
\hline \multirow{2}{*}{$\begin{array}{l}\text { Mosclean trap indoors vs } \\
\text { Mosclean trap outdoors }\end{array}$} & \multirow[t]{2}{*}{ An. funestus } & Mosclean trap outdoor & 13 & $0(0-0.25)$ & Ref & Ref \\
\hline & & Mosclean trap indoor & 91 & $1.5(1-4.25)$ & $6.93(3.85-12.46)$ & $<0.001$ \\
\hline \multirow{2}{*}{$\begin{array}{l}\text { Mosclean trap indoors vs } \\
\text { Mosclean trap outdoors }\end{array}$} & \multirow[t]{2}{*}{ Culex spp. } & Mosclean trap outdoor & 1035 & $24.5(16.75-60)$ & Ref & Ref \\
\hline & & Mosclean trap indoor & 2645 & $69.5(40-131.25)$ & $2.53(1.71-3.75)$ & $<0.001$ \\
\hline \multirow{4}{*}{$\begin{array}{l}\text { Outdoor tests to compare } \\
\text { four trap types }\end{array}$} & \multirow[t]{4}{*}{ An. arabiensis } & BG-sentinel trap & 14 & $0(0-1)$ & Ref & Ref \\
\hline & & Mosclean trap & 65 & $1(0-2.25)$ & $4.36(1.62-11.72)$ & 0.003 \\
\hline & & Mosclean trap $+\mathrm{CO}_{2}$ & 112 & $1(0-8)$ & $7.42(2.85-19.31)$ & $<0.001$ \\
\hline & & Suna trap & 30 & $0.5(0-2)$ & $2.03(0.75-5.47)$ & 0.163 \\
\hline \multirow{4}{*}{$\begin{array}{l}\text { Outdoor tests to compare } \\
\text { four trap types }\end{array}$} & \multirow[t]{4}{*}{ Culex spp. } & BG-sentinel trap & 1552 & 76.5 (26.5-88) & Ref & Ref \\
\hline & & Mosclean trap & 969 & $29(17.25-44.25)$ & $0.59(0.39-0.91)$ & 0.017 \\
\hline & & Mosclean trap $+\mathrm{CO}_{2}$ & 1991 & $46(23.75-78.25)$ & $1.08(1.70-1.67)$ & 0.698 \\
\hline & & Suna trap & 1711 & $62.5(36.75-98.75)$ & $1.08(0.71-1.63)$ & 0.707 \\
\hline
\end{tabular}

significant difference between mean mosquito counts in tests against HLC (RR: 0.74, 95\% CI: 0.51-1.07, $P=0.113)$.

\section{Non-competitive tests of the Mosclean trap and HLC}

In tests when the Mosclean trap or HLC were used singly inside the chambers on different nights without any competition, the traps caught similar numbers of $A n$. arabiensis per night (RR: 1.1, 95\% CI: 0.67-1.82, $P=0.708$ ).

\section{Tests to directly compare the Mosclean trap to Suna and BG-Sentinel traps}

In the comparative evaluation of the Mosclean trap against $\mathrm{CO}_{2}$-baited BG-Sentinel trap and $\mathrm{CO}_{2}$-baited Suna trap in semi-field conditions, 3214 mosquitoes were recaptured (19.1\% of total released over all experimental nights). The proportion of mosquitoes recaptured by the $\mathrm{CO}_{2}$-baited Suna trap was 35.7\% (1149); $\mathrm{CO}_{2}$-baited Mosclean trap, 30\% (963); $\mathrm{CO}_{2}$-baited BGSentinel trap, 24.2\% (777); and un-baited Mosclean trap, $10.1 \%$ (325). The percentages for each trap were calculated from the total numbers recaptured. The Mosclean trap when baited with additional $\mathrm{CO}_{2}$ caught more mosquitoes compared to the BG-Sentinel trap, although the difference was not statistically significant $(P=0.086)$. On the other hand, the $\mathrm{CO}_{2}$-baited Suna trap caught approximately 1.5 times as many An. arabiensis as the $\mathrm{CO}_{2}$-baited BG-Sentinel trap $(P=0.021)$, but a comparable number to the $\mathrm{CO}_{2}$-baited baited Mosclean trap (Table 1).

\section{Results of field tests to compare trapping efficacies of the Mosclean trap, CDC-light trap, BG-Sentinel trap and Suna trap}

Comparison of efficacies of the Mosclean trap and CDC-light trap for sampling indoor host-seeking mosquitoes

A summary of the field results are provided in Table 2. The Mosclean trap caught approximately the same number of $A n$. arabiensis as the CDC-light trap when the traps were set indoors in different huts (RR: 1.18, 95\% CI: 0.84-1.50, $P=0.242$ ). Recent evidence from these field sites suggest that the Anopheles gambiae complex consists entirely of $A n$. arabiensis [49, 50], thus we hereafter refer to them as such throughout the manuscript. The Mosclean trap caught significantly less An. funestus mosquitoes than the CDC-light trap (RR: $0.62,95 \% \mathrm{CI}: 0.43-0.89, P=0.009)$, but was twice as 
Table 3 Parity and insemination rates of An. arabiensis mosquitoes collected by Mosclean trap and CDC-light trap indoors in the field setting. Table also shows odds ratios (OR)

\begin{tabular}{llllll}
\hline Method & Total no. dissected & $\begin{array}{l}\text { Proportion parous } \\
\%(n)\end{array}$ & $\begin{array}{l}\text { OR (95\% Cl) } \\
(P \text {-value })\end{array}$ & $\begin{array}{l}\text { Proportion } \\
\text { inseminated } \\
\%(n)\end{array}$ & $\begin{array}{l}\text { OR (95\% Cl) } \\
(P \text {-value })\end{array}$ \\
\hline CDC-light trap indoors & 251 & $45.8(115)$ & Ref & $90.4(227)$ & Ref \\
Mosclean trap indoors & 181 & $56.4(102)$ & $\begin{array}{l}2.06(1.24-3.41) \\
(P=0.005)\end{array}$ & $87.8(159)$ & $\begin{array}{l}1.57(0.61-4.07) \\
(P=0.353)\end{array}$ \\
\hline
\end{tabular}

Abbreviation: $n$, total number of parous or total number of inseminated mosquitoes

efficacious in catching Culex mosquitoes (RR: 2.18, 95\% CI: $1.72-2.77, P<0.001)$.

\section{Comparison of performance of the Mosclean trap when used indoors and outdoors}

When the efficacy of Mosclean trap was assessed for outdoor and indoor use, it caught more An. arabiensis, An. funestus and Culex mosquitoes indoors than outdoors (Table 2 and Additional file 1: Figure S2). However, these differences were statistically significant for only An. funestus mosquitoes (RR: 6.93, 95\% CI: 3.8512.46, $P<0.001)$.

\section{Tests to compare field efficacies of the Mosclean trap, BG-Sentinel trap and Suna trap outdoors}

In the $4 \times 4$ Latin square experiments, where all the four trap types were tested outdoors in the villages, the total number of An. arabiensis caught was 221 . Of these, the $\mathrm{CO}_{2}$-baited Mosclean trap caught $50.7 \%$ $(n=112)$, the un-baited Mosclean trap caught $29.4 \%$ $(n=65)$, the $\mathrm{CO}_{2}$-baited Suna trap caught $15.6 \%$ $(n=30)$ and the $\mathrm{CO}_{2}$-baited BG-Sentinel trap caught $6.3 \%(n=14)$ over the 20 test nights. The number of $A n$. arabiensis caught by the un-baited Mosclean trap was four times more than the $\mathrm{CO}_{2}$-baited BG-Sentinel $(P=0.003)$, and this increased to seven times when $\mathrm{CO}_{2}$ was added to the Mosclean trap $(P<0.001)$.

Concurrently, 6223 Culex spp. mosquitoes were caught, of which the $\mathrm{CO}_{2}$ baited Mosclean trap caught $32 \%(n=1991), \mathrm{CO}_{2}$-baited Suna trap caught $27.5 \%$ $(n=1711), \mathrm{CO}_{2}$-baited BG-Sentinel trap caught $24.9 \%$ $(n=1552)$ and un-baited Mosclean trap caught $15.6 \%$ $(n=969)$. For this genus, there were no significant differences between the traps (Table 2).

\section{Proportions of parous and inseminated female $A n$.}

\section{arabiensis in Mosclean trap and CDC-light trap catches}

The proportion of parous An. arabiensis females was slightly higher in Mosclean trap collections than CDClight trap collections, while the proportions inseminated were similar. Of the $181 \mathrm{An}$. arabiensis caught by Mosclean trap, which were dissected, $56.4 \%$ were parous and $87.8 \%$ were inseminated (Table 3). Of the $251 A n$. arabiensis caught by CDC-light trap, which were dissected, $45.8 \%$ were parous and $90.4 \%$ were inseminated.

\section{Discussion}

To improve surveillance strategies against vector-borne infections, new trapping devices are required that demonstrate high levels of efficacy, field robustness, affordability and scalability. This study evaluated a new LED trap that emits UV light as the primary mosquito attractant. The trap was compared to existing trapping methods including CDC-light traps with incandescent light bulbs, as is commonly used for trapping mosquitoes inside human dwellings. Other traps tested were the BGSentinel trap and Suna trap. The tests were conducted both indoors and outdoors, in both semi-field settings against laboratory-reared mosquitoes and field settings against wild mosquito populations. Although the main target was the malaria vector $A n$. arabiensis, the field tests also revealed moderate to high efficiencies against other mosquito species. Overall, the Mosclean trap was at least as efficacious as HLC, and twice as efficacious as the CDC-light trap when used indoors for sampling the malaria vectors in the semi-field, although the difference not being statistically significant. Similarly, in the field settings, the trap caught slightly more An. arabiensis than CDC-light trap. For Culex mosquitoes, the Mosclean trap also caught twice as many as the CDC-light trap.

Mosquito traps employ odor and visual stimuli to activate and attract mosquitoes from a distance [17, 51-54]. Insects typically visualize objects in the ultraviolet, blue and green wavelengths [55]. The Mosclean trap was developed to exploit host-seeking behavior of mosquitoes in relation to both olfactory and visual stimuli. The trap utilizes titanium dioxide $\left(\mathrm{TiO}_{2}\right)$ which, releases $\mathrm{CO}_{2}$ by a photocatalyst reaction during the operation. It also emits optimized high efficiency UV LEDs (wavelength of $365 \mathrm{~nm}$ ) to attract mosquitoes. An additional advantage is that the lamp can run for more than 10,000 hours and therefore requires less frequent replacements than the 
incandescent lamps used on the CDC-light trap which typically runs for 1200 hours or less [56].

Previous trials in South Korea had also showed that LED traps caught significantly higher numbers of hostseeking mosquitoes compared to traditional light traps [57]. This may indicate the importance of natural human cues, and also host biomass, which directly influence biting densities [58]. In our outdoor-indoor tests, the traps were set near volunteer-occupied mosquito nets. However, there was likely a greater concentration of host cues indoors due to a higher number of people indoors compared to outdoors, resulting in a higher number of catches indoors. In tests where the Mosclean trap was placed either indoors or outdoors, An. funestus mosquitoes appeared highly endophilic, just as previously observed by Ngowo et al. [50]. The trap was more efficacious indoors than outdoors for catching this vector species, which now mediates more than $80 \%$ of transmission in the study area [49]. Nearly seven times the number of An. funestus and nearly two times the number of An. arabiensis were caught indoors compared to outdoors.

In this study, the Mosclean trap was also evaluated against HLCs in the semi-field, but not in the field due to ethical concerns. In the semi-field tests with laboratoryreared An. arabiensis, the trap was as effective as HLCs in both competitive and non-competitive settings (Table 1). The HLC method has multiple disadvantages which limit its application for field studies. It is labor-intensive and needs close supervision, is expensive and may expose human volunteers to potentially-infectious bites [16]. To address these issues, innovative methods have been developed as substitutes, but many of these alternatives do not completely address the challenges. For example, mosquitoes trapped by the mosquito electrocuting trap (MET) dry quickly, making it unsuitable for scientific investigations, such as dissection for parity and insemination status, which require fresh samples [17]. In Burkina Faso [53], a human decoy trap (HDT) was recently demonstrated to catch significantly higher numbers of Anopheles spp., Culex spp. and Mansonia spp. than HLC. However, HDTs are not easily scalable as they need human volunteers and a supply of boiled water [53], making them expensive to conduct.

The Mosclean trap was also efficient outdoors for trapping An. arabiensis mosquitoes, compared to other trapping devices previously used for the same purpose (Table 2). It caught more malaria vectors and had higher trapping efficiencies, which were further enhanced when $\mathrm{CO}_{2}$ was added. The Suna trap has been observed to perform well in catching An. gambiae in semi-field settings [22] and An. funestus in field settings [39], and is therefore considered an effective field trapping technique. In this study, the field-trapping efficiencies of the Mosclean trap exceeded both the BG-Sentinel trap and Suna trap.
Anopheles arabiensis mosquitoes caught by the traps were dissected to assess epidemiological importance. This examination revealed that more than half of hostseeking mosquitoes caught by Mosclean trap indoors were parous. The values are comparable to or slightly higher than those obtained in CDC light traps (Table 3). Furthermore, nearly $90 \%$ of the females caught indoors were inseminated. Together, these findings suggest that the physiological status, and approximate age of the collected mosquitoes is likely to be similar between the Mosclean trap and CDC light trap. Past studies have shown similar observations of high parity rates among indoor malaria vectors [59].

The version of Mosclean traps used was less expensive than other commercially available traps. For example, the Suna trap and BG-Sentinel trap are listed by the manufacturer at US\$168.11 per unit [60], while the CDC-light trap is listed at US\$ 106.00 per unit [61]. By comparison, the Mosclean trap is available for US\$ 67.05 [62]. Because of their higher energy efficiency, Mosclean traps are also expected to use less battery power than the other traps.

One limitation is that the UV-LED technology may trap other non-target organisms when used in field settings, especially outdoors, potentially making the trap less environmentally friendly. Another limitation is that we did not measure the quantities of $\mathrm{CO}_{2}$ gas produced by the $\mathrm{TiO}_{2}$ component of the Mosclean trap as claimed by the manufacturer. These issues need to be investigated before the trap can be considered as a replacement in regular surveillance programs.

\section{Conclusions}

The UV LED trap (Mosclean trap) demonstrated substantive efficacies for trapping of Anopheles and Culex mosquitoes, and was better indoors than outdoors. The trap was either comparable with or better than existing comparator traps, and could have potential for mosquito surveillance. It is easier to use, cost-friendly and less noisy during its operation. The trap can also be used indoors near human-occupied bednets (the human acting as non-exposed bait) or outdoors, in which case it is beneficial to add other baits such as $\mathrm{CO}_{2}$ gas. Importantly, the Mosclean trap catches mosquito populations of high epidemiological importance, with high proportions of parous and insemination females. However, the potential of LED traps, including the Mosclean trap, to catch non-targeted organisms should be investigated further, especially when used outdoors. Furthermore, more studies should be undertaken to validate these findings in other areas and to assess the efficacy of the Mosclean trap against a greater diversity of mosquito species. 


\section{Supplementary information}

Supplementary information accompanies this paper at https://doi. org/10.1186/s13071-019-3673-7.

\begin{abstract}
Additional file 1: Figure S1. Number of An. arabiensis mosquitoes recaptured per night in the semi-field experiments to evaluate efficacy of the Mosclean trap against other traps. a Mosclean trap tested competitively against CDC light trap indoors, with both traps in the chamber on the same nights. b Mosclean trap tested competitively against HLC when both traps are in the chamber on same nights. c Mosclean trap tested against HLC, when the traps are set in the chambers individually in different nights. Figure S2. Median number of mosquitoes caught per night indoors and outdoors by the Mosclean trap in rural south-eastern Tanzania. Data collected in four houses in 2 villages, over 12 nights for An. arabiensis (a) and Culex spp. (b) mosquitoes
\end{abstract}

\section{Abbreviations}

HLC: human landing catch; LEDs: light-emitting diodes; UV LED: ultraviolet light-emitting diodes; MET: mosquito electrocuting trap; HDT: human decoy trap; $\mathrm{CO}_{2}$ : carbon dioxide.

\section{Acknowledgements}

A special thanks to volunteers Mr Steven Kipande, Mr Andrew Kafwenji and Mr Joseph Kipande for collection of mosquitoes in the semi-field experiments. We also express our deepest gratitude to the administrative team at the Ifakara Health Institute, particularly Mwajabu Hamis, Serbard Mteteleka and Rukiyah Mohammad for their assistance in logistic management. We thank Alex J. Limwagu for map preparation and Eom Hoonsik for reviewing the final manuscript and verifying technical details of the Mosclean trap. We thank the communities in Ulanga District for allowing and supporting us to conduct all field experiments.

\section{Authors' contributions}

EPM and FOO designed the study and developed research protocol. EPM, HSN and FOO analyzed the data and wrote the manuscript. SAM, ASM, EWK and KK conducted the experiments and data collection. EPM, HSN, ASM, SAM and FOO reviewed and revised the manuscript. All authors read and approved the final manuscript.

\section{Funding}

This work was funded by Wellcome Trust Intermediate Fellowship in Public Health and Tropical Medicine grant awarded to FO (Grant Number WT102350/Z/13/Z).

\section{Availability of data and materials}

Data supporting the conclusions of this article are included within the article and its additional files. The dataset generated during this study is available from the corresponding author upon request.

\section{Ethics approval and consent to participate}

This study was approved by the Ifakara Health Institute Review board (Ref. IHI/IRB/EXT/No: 005 - 2018) and the Medical Research Coordinating Council at the Tanzanian National Institute of Medical Research (NIMR) (Ref. NIMR/ HQ/R.8C/Nol.II/880). Written and oral informed consent was obtained from all volunteers participating in this study, after being provided with all necessary information regarding goals, objective, benefits as well as risks associated with the study. In the field experiments, every house selected for mosquito sampling was provided with a new LLIN. Permission was also obtained from community leaders prior to starting the studies.

\section{Consent for publication}

Permission to publish this work was obtained from NIMR (Ref. NIMR/HQ/P.12 VOL XXVI/77).

\section{Competing interests}

The authors declare that they have no competing interests.

\section{Author details}

${ }^{1}$ Environmental Health and Ecological Sciences Department, Ifakara Health Institute, Morogoro, Tanzania. ${ }^{2}$ Institute of Biodiversity, Animal Health and Comparative Medicine, University of Glasgow, Glasgow, UK. ${ }^{3}$ School of Public Health, University of Witwatersrand, Johannesburg, South Africa.

Received: 25 March 2019 Accepted: 17 August 2019

Published online: 27 August 2019

\section{References}

1. WHO. Global technical strategy for malaria 2016-2030. 2015. http://apps. who.int/iris/bitstream/10665/176712/1/9789241564991_eng.pdf?ua=1. Accessed 2 Feb 2019

2. WHO. Global vector control response 2017-2030. Geneva: World Health Organization; 2017

3. Smith DL, McKenzie FE, Snow RW, Hay SI. Revisiting the basic reproductive number for malaria and its implications for malaria control. PLoS Biol. 2007;5:e42.

4. WHO. Malaria surveillance, monitoring \& evaluation: a reference manual. 2018. http://www.who.int/malaria/publications/atoz/9789241565578/ en/. Accessed 5 Feb 2019.

5. Mboera LEG. Sampling techniques for adult Afrotropical malaria vectors and their reliability in the estimation of entomological inoculation rate. Tanzan J Health Res. 2006:7:117-24.

6. Davis JR, Hall T, Chee EM, Majala A, Minjas J, Shiff CJ. Comparison of sampling Anopheline mosquitoes by light-trap and human-bait collections indoors at Bagamoyo, Tanzania. Med Vet Entomol. 1995;9:249-55.

7. Silver JB, Service MW. Mosquito ecology: Field sampling methods. London: Springer; 2008

8. Rapley LP, Johnson PH, Williams CR, Silcock RM, Larkman M, Long SA, et al. A lethal ovitrap-based mass trapping scheme for dengue control in Australia: II. Impact on populations of the mosquito Aedes aegypti. Med Vet Entomol. 2009:23:303-16.

9. Day JF, Sjogren RD. Vector control by removal trapping. Am J Trop Med Hyg. 1994;50:126.

10. Okumu FO, Govella NJ, Moore SJ, Chitnis N, Killeen GF. Potential benefits, limitations and target product-profiles of odor-baited mosquito traps for malaria control in Africa. PLoS One. 2010:5:e11573.

11. Mboera LEG. Sampling techniques for adult Afrotropical malaria vectors and their reliability in the estimation of entomological inoculation rate. Tanzan Health Res Bull. 2005;7:117-24.

12. Canyon DV, Hii JLK. Efficacy of carbon dioxide, 1-octen-3-ol, and lactic acid in modified Fay-Prince traps as compared to man-landing catch of Aedes aegypti. J Am Mosq Control Assoc. 1997:13:66-70.

13. Mathenge E, Misiani GO, Oulo DO, Irungu LW, Ndegwa P, Smith TA, et al. Comparative performance of the Mbita trap, CDC light trap and the human landing catch in the sampling of Anopheles arabiensis, An. funestus and culicine species in a rice irrigation scheme in western Kenya. Malar J. 2005:4:7.

14. Sikulu M, Govella NJ, Ogoma SB, Mpangile J, Kambi SH, Kannady K, et al. Comparative evaluation of the Ifakara tent trap-B, the standardized resting boxes and the human landing catch for sampling malaria vectors and other mosquitoes in urban Dar es Salaam, Tanzania. Malar J. 2009:8:197.

15. Krockel U, Rose A, Eiras AE, Geier M. New tools for surveillance of adult yellow fever mosquitoes: comparison of trap catches with human landing rates in an urban environment. J Am Mosq Control Assoc. 2006;22:229-38.

16. Vector control for malaria and other mosquito-borne diseases. Report of a WHO study group. World Health Organ Tech Rep Ser. 1995;857:1-91.

17. Govella NJ, Maliti DF, Mlwale AT, Masallu JP, Mirzai N, Johnson PCD, et al. An improved mosquito electrocuting trap that safely reproduces epidemiologically relevant metrics of mosquito human-feeding behaviours as determined by human landing catch. Malar J. 2016;15:465

18. Tangena JAA, Thammavong P, Hiscox A, Lindsay SW, Brey PT. The human-baited double net trap: An alternative to human landing catches for collecting outdoor biting mosquitoes in Lao PDR. PLoS One. 2015:10:e0138735.

19. Sudia WD, Chamberlain RW. Battery-operated light trap, an improved model. Mosq News. 1962;22:126-9. 
20. Mboera LE, Kihonda J, Braks MA, Knols BG. Short report: Influence of centers for disease control light trap position, relative to a humanbaited bed net, on catches of Anopheles gambiae and Culex quinquefasciatus in Tanzania. Am J Trop Med Hyg. 1998;59:595-6.

21. Zaim M, Ershadi MR, Manouchehri AV, Hamdi MR. The use of CDC light traps and other procedures for sampling malaria vectors in southern Iran. J Am Mosq Control Assoc. 1986;2:511-5.

22. Hiscox A, Otieno B, Kibet A, Mweresa CK, Omusula P, Geier M, et al. Development and optimization of the Suna trap as a tool for mosquito monitoring and control. Malar J. 2014;13:257.

23. Maciel-de-Freitas R, Eiras ÁE, Lourenço-de-Oliveira R. Field evaluation of effectiveness of the BG-Sentinel, a new trap for capturing adult Aedes aegypti (Diptera: Culicidae). Mem Inst Oswaldo Cruz. 2006;101:321-5.

24. Govella NJ, Chaki PP, Geissbuhler Y, Kannady K, Okumu F, Charlwood JD, et al. A new tent trap for sampling exophagic and endophagic members of the Anopheles gambiae complex. Malar J. 2009:8:157.

25. Williams CR, Long SA, Russell RC, Ritchie SA. Field efficacy of the BGSentinel compared with CDC Backpack Aspirators and CO2-baited EVS traps for collection of adult Aedes aegypti in Cairns, Queensland, Australia. J Am Mosq Control Assoc. 2006:22:296-300.

26. Pombi M, Guelbeogo WM, Calzetta M, Sagnon N, Petrarca V, La Gioia $\checkmark$, et al. Evaluation of a protocol for remote identification of mosquito vector species reveals BG-Sentinel trap as an efficient tool for Anopheles gambiae outdoor collection in Burkina Faso. Malar J. 2015;14:161.

27. Okumu FO, Killeen GF, Ogoma S, Biswaro L, Smallegange RC, Mbeyela E, et al. Development and field evaluation of a synthetic mosquito lure that Is more attractive than humans. PLoS One. 2010;5:e8951.

28. Mukabana WR, Mweresa CK, Otieno B, Omusula P, Smallegange RC, van Loon JJA, et al. A novel synthetic odorant blend for trapping of malaria and other African mosquito species. J Chem Ecol. 2012;38:235-44.

29. Costa-Neta BM, da Silva AA, Brito JM, Moraes JLP, Rebêlo JMM, Silva FS. Light-emitting diode (LED) traps improve the light-trapping of anopheline mosquitoes. J Med Entomol. 2017:54:1699-703.

30. Conhstaedt L, Gillen Jl, Munstermann LE. Light-emitting diode technology improves insect trapping. J Am Mosq Control Assoc. 2008;24:331-4.

31. Silva FS, Da Silva AA, Rebelo JMM. An evaluation of light-emitting diode (LED) traps at capturing phlebotomine sand flies (Diptera: Psychodidae) in a Livestock area in Brazil. J Med Entomol. 2016;53:634-8.

32. Hoel DF, Butler JF, Fawaz EY, Watany N, El-Hossary SS, Villinski J. Response of phlebotomine sand flies to light-emitting diode-modified light traps in southern Egypt. J Vector Ecol. 2007;32:302-8.

33. Burkett DA, Butler JF, Kline DL. Field evaluation of colored light-emitting diodes as attractants for woodland mosquitoes and other diptera in north central Florida. J Am Mosq Control Assoc. 1998;14:186-95.

34. Seoul Viosys. Mosclean trap. 2017. http://www.seoulviosys.com/en/ solutions/. Accessed 5 Feb 2019.

35. Ferguson HM, Ng'habi KR, Walder T, Kadungula D, Moore SJ, Lyimo I, et al. Establishment of a large semi-field system for experimental study of African malaria vector ecology and control in Tanzania. Malar J. 2008;7:158.

36. Okumu FO, Moore J, Mbeyela E, Sherlock M, Sangusangu R, Ligamba $G$, et al. A modified experimental hut design for studying responses of disease-transmitting mosquitoes to indoor interventions: the Ifakara experimental huts. PLoS One. 2012;7:e30967.

37. Batista EPA, Ngowo HS, Opiyo M, Shubis GK, Meza FC, Okumu FO, et al. Semi-field assessment of the BG-Malaria trap for monitoring the African malaria vector, Anopheles arabiensis. PLoS One. 2017;12:e0186696.

38. Farajollahi A, Kesavaraju B, Price DC, Williams GM, Healy SP, Gaugler R, et al. Field efficacy of BG-Sentinel and industry-standard traps for Aedes albopictus (Diptera: Culicidae) and West Nile virus surveillance. J Med Entomol. 2009;46:919-25

39. Homan T, Hiscox A, Mweresa CK, Masiga D, Mukabana WR, Oria P, et al. The effect of mass mosquito trapping on malaria transmission and disease burden (SolarMal): a stepped-wedge cluster-randomised trial. Lancet. 2016;338:1193-201.

40. Mburu MM, Zembere K, Hiscox A, Banda J, Phiri KS, van den Berg H, et al. Assessment of the Suna trap for sampling mosquitoes indoors and outdoors. Malar J. 2019;18:51

41. Batista EPA, Eiras AE, Ngowo H, Opiyo M, Shubis GK, Meza FC, et al. Field evaluation of the BG-Malaria trap for monitoring malaria vectors in rural Tanzanian villages. PLoS One. 2018;13:e0205358.
42. Mweresa CK, Omusula P, Otieno B, Van Loon JJA, Takken W, Mukabana WR. Molasses as a source of carbon dioxide for attracting the malaria mosquitoes Anopheles gambiae and Anopheles funestus. Malar J. 2014;13:160.

43. Mmbando AS, Okumu FO, Mgando JP, Sumaye RD, Matowo NS, Madumla $E$, et al. Effects of a new outdoor mosquito control device, the mosquito landing box, on densities and survival of the malaria vector, Anopheles arabiensis, inside controlled semi-field settings. Malar J. 2015;14:494.

44. Detinova TS. Age-grouping methods in Diptera of medical importance with special reference to some vectors of malaria. Monogr Ser World Health Organ. 1962;47:13-191.

45. Rosay B. Anatomical indicators for assessing age of mosquitoes: Dissection techniques and field application of methods. Mosq News. 1969;29:419-23.

46. R Development Core Team. R: a language and environment for statistical computing. Vienna: R Foundation for Statistical Computing; 2018.

47. Bates D, Maechler M. Package 'Ime4'(Version 1.1-21): linear mixedeffects models using S4 classes. 2009. http//cran.r-project.org/web/ packages/Ime4/Ime4.pdf. Accessed 5 Mar 2019.

48. Wickham H. ggplot2. New York: Springer-Verlag; 2009.

49. Kaindoa EW, Matowo NS, Ngowo HS, Mkandawile G, Mmbando A, Finda $M$, et al. Interventions that effectively target Anopheles funestus mosquitoes could significantly improve control of persistent malaria transmission in south-eastern Tanzania. PLoS One. 2017;12:e0177807.

50. Ngowo HS, Kaindoa EW, Matthiopoulos J, Ferguson HM, Okumu FO. Variations in household microclimate affect outdoor-biting behaviour of malaria vectors. Wellcome Open Res. 2017;2:102

51. Mboera LEG, Knols BGJ, Braks MAH, Takken W. Comparison of carbon dioxide-baited trapping systems for sampling outdoor mosquito populations in Tanzania. Med Vet Entomol. 2000;14:257-63.

52. Matowo NS, Moore J, Mapua S, Madumla EP, Moshi IR, Kaindoa EW, et al. Using a new odour-baited device to explore options for luring and killing outdoor-biting malaria vectors: a report on design and field evaluation of the Mosquito Landing Box. Parasit Vectors. 2013;6:137.

53. Hawkes FM, Dabiré RK, Sawadogo SP, Torr SJ, Gibson G. Exploiting Anopheles responses to thermal, odour and visual stimuli to improve surveillance and control of malaria. Sci Rep. 2017;7:17283.

54. Gillies MT, Wilkes TJ. The range of attraction of animal baits and carbon dioxide for mosquitoes. Studies in a freshwater area of West Africa. Bull Entomol Res. 1972;61:389-404.

55. Briscoe $A D$, Chittka L. The evolution of color vision in insects. Annu Rev Entomol. 2001;46:471-510.

56. Viribright. Comparing LED vs CFL vs incandescent light bulbs. 2019. https://www.viribright.com/lumen-output-comparing-led-vs-cf-vsincandescent-wattage/. Accessed 10 Feb 2019.

57. Kim HC, Kim MS, Choi KS, Hwang DU, Johnson JL, Klein TA. Comparison of adult mosquito black-light and light-emitting diode traps at three cowsheds located in malaria-endemic areas of the Republic of Korea. J Med Entomol. 2017;54:221-8.

58. Kaindoa EW, Mkandawile G, Ligamba G, Kelly-Hope LA, Okumu FO. Correlations between household occupancy and malaria vector biting risk in rural Tanzanian villages: Implications for high-resolution spatial targeting of control interventions. Malar J. 2016;15:199.

59. Brady J. Results of age-grouping dissection on four species of Anopheles from southern Ghana. Bull World Health Organ. 1963;29:147-53.

60. Biogents. The BG-Suna for professionals is specifically developed against malaria mosquitoes. 2019. https://eu.biogents.com/bgsuna/\#1483546552203-7d9aa172-8e99. Accessed 5 Feb 2019.

61. John W. Hock. CDC Miniature Light Trap. 2019. https://johnwhock.com/ products/mosquito-sandfly-traps/cdc-miniature-light-trap/. Accessed 5 Feb 2019.

62. McQwin's wholesale. Mosclean mosquitoes trap. 2019. https://www. mqsupplies.com/mosclean-insect-trap/indoor-1/KEE-O-Mosclean-IW1. Accessed 11 Aug 2019.

\section{Publisher's Note}

Springer Nature remains neutral with regard to jurisdictional claims in published maps and institutional affiliations. 